Effective in 2014, the CPD programme for SAMJ will be administered by Medical Practice Consulting: CPD questionnaires must be completed online at www.mpconsulting.co.za

True (A) or false (B):

\section{The safety of osteoporosis medication}

1. Osteoporosis is a common, costly and serious disease with $1 / 5$ patients dying within 1 year following a hip fracture.

2. Treatment of osteoporosis with calcium and vitamin $\mathrm{D}$ is associated with an impressive reduction in fracture risk.

3. In the absence of contraindications, the use of menopausal hormone (oestrogen without or without progestin) therapy in women aged 50 - 60 years is safe and appropriate to manage osteoporosis, and is the drug of choice if menopausal symptoms are present

The risks of gastrointestinal injury due to ingested magnetic beads

4. When multiple individual magnets are ingested, they can conglomerate in different segments of bowel causing pressure necrosis, perforation and/or fistula formation anywhere along the gastrointestinal tract.

Acute porphyria

5. The symptomatic patient with an acute, severe porphyria typically presents with a sudden onset of a characteristic syndrome of acute abdominal pain, which may progress to a sudden onset of quadriparesis over the course of a few hours.

\section{High prevalence of cisplatin-induced ototoxicity}

6. Cisplatin is one of the most widely used and effective cytotoxic agents currently available for the treatment of soft-tissue cancers, with a reported cure rate of up to $85 \%$.

7. Cisplatin-induced ototoxicity is dependent on age at initiation of treatment, with those $<5$ years and those $>40$ years of age being most susceptible.

Angiotensin converting enzyme inhibitors (ACEIs) v. angiotensin receptor blockers (ARBs) in the management of hypertension

8. Hypertension is the most prevalent chronic disease among medical aid members, affecting $\sim 12 \%$.

9. In the drug utilisation review process, managed-care companies should continue to recommend ACEIs, rather than ARBs, in the treatment of their hypertension patients.
Mammographic screening for breast cancer in a resource-restricted environment

10. After carcinoma of the cervix, breast cancer is the second most common female cancer in South Africa.

\section{Hydrocephalus in spina bifida}

11. More than $80 \%$ of those who develop hydrocephalus ultimately require a surgical procedure to treat the condition.

12. Daily clinical assessment of hydrocephalus involves examination of the fontanelle and measurement of the head circumference.

13. Once a shunt has been placed, the later development of symptoms or signs of Chiari malformation or syringomyelia should raise suspicion of shunt failure.

\section{Occult spinal dysraphism}

14. In occult spinal dysraphism, spinal lipomas and dermal sinus tracks are the most common disorders presenting as clinical problems.

15. About $50-86 \%$ of patients with occult spinal dysraphism will have cutaneous stigmata.

16. If a patient has an anorectal malformation it is routine to perform spinal magnetic resonance imaging to exclude an associated tethered spinal cord.

The orthopaedic management of myelomeningocele

17. The primary goal of the orthopaedic management of myelomeningocele is mobility.

18. In the paraplegic group, spinal scoliosis usually develops by the age of 10 years.

Beyond the operating theatre: Long-term quality of life in spina bifida

19. Incontinence of urine is managed effectively using a regimen of 3-hourly self-catheterisation, daily bowel washout and anticholinergic drugs three times per day.

20. The potential for pressure sores is increased in the pelvic area by urinary incontinence.

A maximum of 3 CEUs will be awarded per correctly completed test.

INSTRUCTIONS

1. Read the journal. All the answers will be found there. 2. Go to www.mpconsulting.co.za to answer the questions.

Accreditation number: MDB001/007/01/2014 (Clinical)

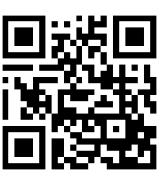

